\title{
SOME RESULTS FOR BOREL TRANSFORMS
}

\author{
J. P. KING
}

1. Introduction. Let $\lambda=\left\{\lambda_{p}\right\}$ be a sequence of complex numbers and let the matrix $A(\lambda)=\left(a_{n k}\right)$ be defined by

$$
\begin{aligned}
& a_{0 k}=0, \quad k=0,1,2, \cdots \\
& \prod_{p=1}^{n} \exp \left(\lambda_{p}(z-1)\right)=\sum_{k=0}^{\infty} a_{n k} z^{k} .
\end{aligned}
$$

In the special case $\lambda=\delta=(1,1,1, \cdots)$, the elements of $A(\lambda)=A(\delta)$ reduce to $a_{n k}=e^{-n} n^{k} / k$ ! The matrix $A(\delta)$ is the discrete Borel sequenceto-sequence transform which has been studied by Gaier [3] and Meyer-König and Zeller [7].

In $\$ 2$ the regularity of the matrix $A(\lambda)$ is investigated and a theorem concerning the summability of Taylor series by $A(\lambda)$ is considered. In $\$ 3$ the $A(\lambda)$-summability of series of Legendre polynomials is studied. These results are believed to be new even in the case $\lambda=\delta$. In $\$ 4$ a theorem concerning the approximation properties of a class of positive linear operators, defined on $C[0, b]$ which are associated with $A(\lambda)$ is proved.

These operators are generalizations of operators which have been studied by Szasz [8], Cheney and Sharma [1], and G. M. Mirakyan $[5$, p. 53].

\section{Regularity of $A(\lambda)$.}

Theorem 1. If $\sum_{p=1}^{n} \lambda_{p} \geqq 0$ for each $n=1,2, \cdots$, and $\sum_{p=1}^{\infty} \lambda_{p}=\infty$ then $A(\lambda)$ is regular.

Proof. It is clear that $\sum_{\mathbf{k}=0}^{\infty} a_{n k}=1$. Since

$$
a_{n k}=\frac{1}{k !} \exp \left(-\sum_{p=1}^{n} \lambda_{p}\right) \cdot\left(\sum_{p=1}^{n} \lambda_{p}\right)^{k}, \quad k, n=1,2, \cdots,
$$

it is clear that $a_{n k} \geqq 0$ for $k, n=0,1,2, \cdots$, and $\lim _{n \rightarrow \infty} a_{n k}=0$ for $k=0,1,2, \cdots$. The Silverman-Toeplitz conditions are, therefore, satisfied. Hence $A(\lambda)$ is regular.

Theorem 2. If $\lambda_{p}$ is real for $p=1,2, \cdots$ and $\sum_{p=1}^{\infty} \lambda_{p}=\infty$ then the sequence of partial sums of the geometric series is $A(\lambda)$-summable to $1 /(1-z)$ in the half-plane $H=\{z: \operatorname{Re}(z)<1\}$. The summability is uniform on each compact subset of $H$.

Received by the editors April 12, 1967. 
Proof. Let $\left\{\sigma_{n}\right\}$ denote the $A$-transform of the sequence of partial sums of the geometric series. Let

$$
R=\left\{z: \lim _{n \rightarrow \infty} \sigma_{n}=1 /(1-z)\right\}
$$

It is easy to see that

$$
R=\left\{z: \lim _{n \rightarrow \infty} \sum_{k=0}^{\infty} a_{n k} z^{k}=0\right\}
$$

But

$$
\sum_{k=0}^{\infty} a_{n k} z^{k}=\prod_{p=1}^{n} \exp \left(\lambda_{p}(z-1)\right)
$$

for $n=1,2, \cdots$. Hence

$$
\left|\sum_{k=0}^{n} a_{n k} z^{k}\right|=\exp \left(\operatorname{Re}(z-1) \sum_{p=1}^{n} \lambda_{p}\right) .
$$

So $z \in R$ if and only if $\operatorname{Re}(z)<1$. Hence $R=H$.

Theorem 3. Let $\lambda_{p}$ be real for $p=1,2, \cdots$ and let $\sum_{p=1}^{\infty} \lambda_{p}=\infty$. If $\sum a_{n} z^{n}$ is a Taylor series with nonzero radius of convergence then the sequence of partial sums is $A(\lambda)$-summable to the value of the function defined by the series in the Borel polygon of the function.

Proof. The proof is a straightforward application of Okada's theorem [6, p. 119].

3. Summability of series of Legendre polynomials. Let $P_{n}$ and $Q_{n}$ denote, respectively, the $n$th Legendre polynomial of first and second kind. $P_{n}$ and $Q_{n}$ are determined by [9, Chapter XV],

$$
P_{n}=\frac{1}{\pi} \int_{0}^{\pi}[\zeta(\phi)]^{n} d \phi
$$

and

$$
Q_{n}=\int_{0}^{\infty}[\tau(u)]^{-n-1} d u,
$$

where $\zeta(\phi)=z+\left(z^{2}-1\right)^{1 / 2} \cos \phi$ and $\tau(u)=t+\left(t^{2}-1\right)^{1 / 2} \cosh u$. (The branch of $\left(z^{2}-1\right)^{1 / 2}$ is chosen so that $z+\left(z^{2}-1\right)^{1 / 2}$ lies outside the unit circle.)

Let $\left\{s_{n}(z, t)\right\}$ be defined by

$$
s_{n}(z, t)=\sum_{k=0}^{n}(2 k+1) P_{k}(z) Q_{k}(t), \quad n=0,1, \cdots .
$$


It is known [9, Chapter XV] that $\left\{s_{n}(z, t)\right\}$ converges to $1 /(t-z)$ if $t$ is in the interior of the ellipse with foci \pm 1 which passes through $t$. The following theorem shows that, under certain conditions, $\left\{s_{n}(z, t)\right\}$ is $A(\lambda)$-summable to $1 /(t-z)$ in a larger region.

THEOREM 4. Let $\lambda_{p}$ be real for $p=1,2, \cdots$ and $\sum_{p=1}^{\infty} \lambda_{p}=\infty$. Let $0<\alpha<1,0<\beta$ and let

$$
\begin{aligned}
& S(t, \alpha)=\{z: \operatorname{Re}(\zeta(\phi) / \tau(u))<\alpha, 0 \leqq \phi \leqq \pi, 0 \leqq u\} \\
& T(t, \beta)=\{z:|\zeta(\phi) / \tau(u)|<\beta, 0 \leqq \phi \leqq \pi, 0 \leqq u\}
\end{aligned}
$$

and

$$
R(t, \alpha, \beta)=S(t, \alpha) \cap T(t, \beta) .
$$

Then $\left\{s_{n}(z, t)\right\}$ is $A(\lambda)$-summable to $1 /(t-z)$ if $z \in R(t, \alpha, \beta)$.

Proof. It follows from the Christoffel formula [9, Chapter XV] that

$$
1 /(t-z)=s_{n}(z, t)+(n+1) d_{n}(z, t) /(t-z)
$$

where

$$
d_{n}(z, t)=P_{n+1}(z) Q_{n}(t)-P_{n}(z) Q_{n+1}(t) .
$$

It follows from (2), (3), and (5) that

$$
d_{n}(z, t)=\frac{1}{\pi} \int_{0}^{\pi} \int_{0}^{\infty}\left(\frac{\zeta}{\tau}\right)^{n}\left[\frac{\zeta}{\tau}-\frac{1}{\tau^{2}}\right] d \phi d u .
$$

So if $\left\{\sigma_{n}(z, t)\right\}$ denotes the $A(\lambda)$-transform of $\left\{s_{n}(z, t)\right\}$, it follows from (4), (5) and (6) that

$$
1 /(t-z)=\sigma_{n}(z, t)+h_{n}(z, t) /(t-z),
$$

where

$$
h_{n}(z, t)=\sum_{k=0}^{\infty} a_{n k} \frac{(k+1)}{\pi} \int_{0}^{\pi} \int_{0}^{\infty}\left(\frac{\zeta}{\tau}\right)^{k}\left[\frac{\zeta}{\tau}-\frac{1}{\tau^{2}}\right] d \phi d u .
$$

Hence, $\lim _{n \rightarrow \infty} \sigma_{n}(z, t)=1 /(t-z)$ if $\lim _{n \rightarrow \infty} h_{n}(z, t)=0$.

It is not difficult to see that $\int_{0}^{\pi} \int_{0}^{\infty}\left\{|\zeta / \tau|+\left|1 / \tau^{2}\right|\right\} d \phi d u$ is uniformly bounded for $z \in R(t, \alpha, \beta)$ so that $\int_{0}^{\pi} \int_{0}^{\infty}\left[\zeta / \tau-1 / \tau^{2}\right] d \phi d u$ is uniformly bounded on $R(t, \alpha, \beta)$.

A formal interchange of integration and summation gives

$$
h_{n}(z, t)=\frac{1}{\pi} \int_{0}^{\pi} \int_{0}^{\infty}\left[\frac{\zeta}{\tau}-\frac{1}{\tau^{2}}\right] \sum_{k=0}^{\infty}(k+1) a_{n k}\left(\frac{\zeta}{\tau}\right)^{k} d \phi d u .
$$


A simple calculation then yields

$$
\begin{aligned}
h_{n}(z, t)= & \frac{1}{\pi} \int_{0}^{\pi} \int_{0}^{\infty}\left[\frac{\zeta}{\tau}-\frac{1}{\tau^{2}}\right] \\
& \cdot \prod_{p=1}^{n} \exp \left(\lambda_{p}\left(\frac{\zeta}{\tau}-1\right)\right)\left[1+\frac{\zeta}{\tau} \sum_{p=1}^{n} \lambda_{p}\right] d \phi d u .
\end{aligned}
$$

Let

$$
I_{n}=\prod_{p=1}^{n} \exp \left(\lambda_{p}\left(\frac{\zeta}{\tau}-1\right)\right)\left[1+\frac{\zeta}{\tau} \sum_{p=1}^{n} \lambda_{p}\right] .
$$

It follows that

$$
\left|I_{n}\right|=\exp \left(\operatorname{Re}\left(\frac{\zeta}{\tau}-1\right) \sum_{p=1}^{n} \lambda_{p}\right)\left|1+\frac{\zeta}{\tau} \sum_{p=1}^{n} \lambda_{p}\right| .
$$

Hence $z \in R(t, \alpha, \beta)$ implies $\lim _{n \rightarrow \infty} I_{n}=0$ and therefore $\lim _{n \rightarrow \infty} h_{n}(z, t)$ $=0$. Moreover, if $z \in T(t, \beta)$ the interchange of integration and summation used in the above is valid since the series involved converges uniformly. Therefore, $z \in R(t, \alpha)$ implies $\lim _{n \rightarrow \infty} \sigma_{n}(z, t)=1 /(t-z)$. This completes the proof.

The sets $S(t, \alpha)$ and $T(t, \beta)$ are the same as regions which were studied by Cowling and King [2] in connection with the Lototsky and Taylor summability of series of Legendre polynomials. An analysis identical to that used in [2] gives the following result

THEOREM 5. Let $\lambda_{p}$ be real for $p=1,2, \cdots$ and let $\sum_{p=1}^{\infty} \lambda_{p}=\infty$. Let $0<\alpha<1$ and $0<\beta$. For each $u \geqq 0$, let $H(\tau(u), \alpha)$ be the open halfplane containing the origin whose boundary is the line through the point $\alpha \tau(u)$ which is perpendicular to the line from the origin throngh $\tau(u)$. Let $B(t)$ be the image of $\cap\{H(\tau(u), \alpha): u \geqq 0\}$ under the mapping $t=1 / 2(w+1 / w)$. Let $D(t)$ denote the bounded region whose boundary is the ellipse with foci \pm 1 which passes through the point $1 / 2\left[(\beta+1 / \beta) t+(\beta-1 / \beta)\left(t^{2}-1\right)^{1 / 2}\right]$. Then $\left\{s_{n}(z, t)\right\}$ is $A(\lambda)$-summable to $1 /(t-z)$ whenever $z$ lies in any compact subset of $B(t) \cap D(t)$.

Now let $f$ be analytic on the interval $[-1,1]$ and let $\left\{t_{n}(z)\right\}$ be the sequence of partial sums of the series of Legendre polynomials which represents $f(z)$ in the ellipse with foci \pm 1 which passes through no singular point of $f$. The region in which $\left\{t_{n}(z)\right\}$ is $A(\lambda)$-summable to $f(z)$ may be determined in terms of $B(t) \cap D(t)$ and the distribution of the singular points of $f$ by an analysis identical to that used in [2]. This analysis gives the result 
THEOREM 6. Let $\lambda_{p}$ be real for $p=1,2, \cdots$ and let $\sum_{p=1}^{\infty} \lambda_{p}=\infty$. Let $0<\alpha<1$ and $0<\beta$. Let $B(t)$ and $D(t)$ be as in Theorem 5 and let $V(t)=B(t) \cap D(t)$. The sequence $\left\{t_{n}(z)\right\}$ is $A(\lambda)$-summable to $f(z)$ whenever $z$ lies in any compact subset of $\cap\{V(t): t \in U\}$ where $U$ is the set of all singular points of $f$.

It is shown in [2] that the region $\cap\{V(t): t \in U\}$ contains the ellipse of convergence of $\left\{t_{n}(z)\right\}$. Theorem 6 may also be obtained from Theorem 5 by making use of a general result of Jakimovski's [4].

4. A positive linear operator associated with $A(\lambda)$. Let $b>0$ and let $\lambda_{p}$ be a real valued function defined on the interval $[0, b]$. Let $B$ denote the real valued functions which are bounded on $[0, \infty)$. Let $a_{n k}=a_{n k}(x)$ be defined by:

$$
\begin{aligned}
& a_{0 k}=0, \quad k=0,1,2, \cdots \\
& \prod_{p=1}^{n} \exp \left(\lambda_{p}(x)(z-1)\right)=\sum_{k=0}^{\infty} a_{n k}(x) z^{k} .
\end{aligned}
$$

For each $f \in C[0, b] \cap B$ let

$$
B_{n}(f)(x)=\sum_{k=0}^{\infty} a_{n k}(x) f\left(\frac{k}{n}\right) .
$$

THEOREM 7. Let $\left\{t_{n}(x)\right\}$ denote the $(C, 1)$-transform of the sequence $\left\{\lambda_{p}(x)\right\}$. If $\lim _{n \rightarrow \infty} t_{n}(x)=x$ uniformly on $[0, b]$, and if $\sum_{p=1}^{n} \lambda_{p}(x) \geqq 0$ for each $n=1,2, \cdots$, and $x \in[0, b]$, then $\lim _{n \rightarrow \infty} B_{n}(f)(x)=f(x)$ uniformly on $[0, b]$ for each $f \in C[0, b] \cap B$.

Proof. The hypothesis $\sum_{p=1}^{n} \lambda_{p}(x) \geqq 0$ implies that $a_{n k}(x) \geqq 0$ for each $x \in[0, b]$. Hence $f(x) \geqq 0$ for $x \in[0, b]$ implies that $B_{n}(f)(x) \geqq 0$ for each $x \in[0, b]$. Therefore $B_{n}$ is a positive operator on $C[0, b]$ for each $n=1,2, \cdots$. It is clear that $B_{n}$ is linear for each $n=1,2, \cdots$. According to a theorem of Korovkin [5, pp. 7, 8] it is sufficient to prove that $\lim _{n \rightarrow \infty} B_{n}\left(e^{i}\right)(x)=x^{i}$ uniformly on $[0, b]$ for $i=0,1,2$, where $e^{i}$ is defined by $e^{i}(x)=x^{i}$ for $i=0,1,2$.

It is clear that $B_{n}\left(e^{0}\right)(x)=1$. Let $P_{n}(z)=\prod_{p=1}^{n} \exp \left(\lambda_{p}(x)(z-1)\right)$. It follows from (7) that

$$
P_{n}^{\prime}(z)=\sum_{k=0}^{\infty} k a_{n k}(x) z^{k-1} .
$$

But also

$$
P_{n}^{\prime}(z)=P_{n}(z) \sum_{p=1}^{n} \lambda_{p}(x)
$$


The last two equations, and the fact that $P_{n}(1)=1$, imply

$$
\frac{1}{n} \sum_{k=0}^{\infty} k a_{n k}(x)=\frac{1}{n} \sum_{p=1}^{n} \lambda_{p}(x) \text {. }
$$

Hence $B_{n}\left(e^{1}\right)(x)=t_{n}(x)$. Therefore

$$
\lim _{n \rightarrow \infty} B_{n}\left(e^{1}\right)(x)=x
$$

uniformly on $[0, b]$. It follows from (7) that

$$
P_{n}^{\prime \prime}(z)=\sum_{k=0}^{\infty} k(k-1) a_{n k}(x) z^{k-2},
$$

and from (9) that

$$
P_{n}^{\prime \prime}(z)=\left[\sum_{p=1}^{n} \lambda_{p}(x)\right]^{2} \prod_{p=1}^{n} \exp \left(\lambda_{p}(x)(z-1)\right) .
$$

Therefore

$$
\left[\sum_{p=1}^{n} \lambda_{p}(x)\right]^{2}=\sum_{k=0}^{\infty} k^{2} a_{n k}(x)-\sum_{k=0}^{\infty} k a_{n k}(x)
$$

and hence

$$
B_{n}\left(e^{2}\right)(x)=\frac{1}{n^{2}}\left[\sum_{p=1}^{n} \lambda_{p}(x)\right]^{2}+\frac{1}{n^{2}} \sum_{k=0}^{\infty} k a_{n k}(x) .
$$

Therefore,

$$
B_{n}\left(e^{2}\right)(x)=t_{n}^{2}(x)+t_{n}(x) / n .
$$

Consequently,

$$
\lim _{n \rightarrow \infty} B_{n}\left(e^{2}\right)(x)=x^{2}
$$

uniformly on $[0, b]$. This proves the theorem.

Corollary. Let $\lambda_{p}(x) \geqq 0$ for each $x \in[0, b]$ and $p=1,2, \cdots$. If $\left\{\lambda_{p}(x)\right\}$ converges to $x$ uniformly on $[0, b]$ then $\lim _{n \rightarrow \infty} B_{n}(f, x)=f(x)$ uniformly on $[0, b]$ for each $f \in C[0, b] \cap B$.

Proof. The $(C, 1)$ method is regular and preserves uniform convergence.

In the special case $\lambda_{p}(x)=x, p=1,2, \cdots$, the operators $B_{n}$ reduce to

$$
B_{n}(f)(x)=e^{-n x} \sum_{k=0}^{\infty} \frac{n^{k} x^{k}}{k !} f\left(\frac{k}{n}\right) .
$$


For this choice of $\lambda_{p}(x)$, Theorem 5 gives a result due to Szasz [8] and, evidently, to Mirakyan [5, p. 53].

\section{REFERENCES}

1. E. W. Cheney and A. Sharma, Bernstein power series, Canad. J. Math. 16 (1964), 241-252.

2. V. F. Cowling and J. P. King, On the Taylor and Lototsky summability of series of Legendre polynomials, J. Analyse Math. 10 (1962-63), 139-152.

3. D. Gaier, On modified Borel methods, Proc. Amer. Math. Soc. 6 (1955), 873-879.

4. A. Jakimovski, Analytic continuation and summability of series of Legendre polynomials, Quart. J. Math. Oxford Ser. (2) 15 (1964), 289-302.

5. G. G. Lorentz, Approximation of functions, Holt, New York, 1966.

6. - Bernstein polynomials, Univ. of Toronto Press, Toronto, 1953.

7. W. Meyer-König and K. Zeller, On Borel's method of summability, Proc. Amer. Math. Soc. 11 (1960), 307-314.

8. Otto Szasz, Generalizations of S. Bernstein's polynomials to the infinite interval, J. Res. Nat. Bur. Standards 45 (1950), 239-245; Collected mathematical works, Univ. of Cincinnati, Cincinnati, 1955, pp. 1401-1407.

9. E. T. Whittaker and G. N. Watson, A course of modern analysis, Cambridge Univ. Press, London, 1946.

LEHIGH UNIVERSITY 